\title{
ANTIRADICAL CAPACITY AND POLYPHENOL COMPOSITION OF ASPARAGUS SPEARS VARIETIES CULTIVATED UNDER DIFFERENT SUNLIGHT CONDITIONS
}

\author{
Bartosz Kulczyński, Joanna Kobus-Cisowska ${ }^{\bowtie}$, Dominik Kmiecik, \\ Anna Gramza-Michałowska, Dorota Golczak, Józef Korczak \\ Faculty of Food Science and Nutrition, Poznań University of Life Sciences \\ Wojska Polskiego 31, 60-634 Poznań, Poland
}

\begin{abstract}
Background. Asparagus officinalis has a high nutritional value. Asparagus is rich in a number of bioactive compounds, mainly flavonoids (quercetin), glutathione, vitamin C, vitamin E, fructans (inulin and fructooligosaccharides) and phytosterols (b-sitosterol). These compounds may play an important role in human health. The purpose of this study was to examine the antioxidant potential and polyphenol composition of white, pale-colored and green asparagus spears of different cultivars.

Material and methods. Investigations were conducted on different asparagus spear extracts. The study included three colors of asparagus (white, pale-colored and green) from five different cultivars subjected to the ethanol extraction procedure. Total phenolic content was also determined by the Folin-Ciocalteu method. Polyphenol (phenolic acids and flavonols) composition was estimated using the HPLC method. The antioxidant properties of extracts were examined using DPPH, ABTS and metal ion chelating assays.

Results. The highest contents of phenolic and flavonoids were observed in green asparagus from Grolim and the lowest in pale-colored asparagus from Gyjmlin. It was found that both the color of asparagus and the cultivar had a significant effect on the composition of phenolic acid and flavonols. Radical scavenging activity toward $\mathrm{DPPH}^{\cdot}$ and $\mathrm{ABTS}$ was highest for green asparagus cv. Grolim and Eposs. The greatest number of Fe ions was chelated by samples of green asparagus cv. Grolim and Huchel's Alpha and pale-colored asparagus cv. Huchel's Alpha.

Conclusion. It was shown that the antioxidant activity of asparagus spears measured by antiradical and chelating activity test depends on variety and color. The highest activity was found in green asparagus and the lowest was identified in white asparagus extracts. It has also been clarified that changes in flavonol and phenolic acid composition and increases in their diversity depends on growing with sunlight and variety. Asparagus can provide a valuable source of phenolic compounds in the human diet.
\end{abstract}

Key words: asparagus, antioxidant activity, bioactive compounds, polyphenols, antiradical properties

\section{INTRODUCTION}

In recent years we have been observing an increased consumer interest in plants, which are rich sources of biologically active components and also exhibit antioxidant properties. Among the bioactive components, polyphenols (phenolic acids, flavonoids and tannins) are the most important group as their presence 
is greatest (Gramza-Michalowska et al., 2007). Polyphenolic compounds contribute significantly to the prevention of many civilization-related diseases, such as cancer, cardiovascular diseases, and neurological diseases, such as Parkinson's disease or Alzheimer's disease, and diabetes.

Asparagus (Asparagus officinalis L.) is an interesting vegetable because of its nutritive value and low calorie content. Asparagus of any cultivar may be consumed in three color forms resulting from the cultivation method adopted, i.e. white, pale-colored or green (Fuentes-Alventosa et al., 2013; Wang et al., 2011). Usually the cultivation of asparagus is carried out in the dark (under the ground without sunlight), so white asparagus are the most popular.

The underground part of the plant is called the crown, which is composed of rhizomes. Clusters of buds are found there, while the root system extends laterally. This is where nutrients are stored for the plant. A properly developed root system determines the quality and volume of the crop. Shoots, also called spears, grow from the buds which form on the rhizome after harvest. Spears which are white in color (considered the most valuable), grow underground with no access to light and are consumed as a vegetable. Under the influence of solar radiation, asparagus tips change from pale-colored to the whole asparagus spear turning green (Albanese et al., 2007).

The number of varieties of asparagus is constantly changing. Many of them have also been modified to meet the requirements and expectations of both producers and customers. It is believed that there is no perfect variety of asparagus in all conditions and the differences between them are often not clear (Kohmura et al., 2008).

In terms of nutritional value and antioxidant potential of asparagus, the results of studies on this subject are very diverse. Some publications inform of the high antioxidant activity of asparagus while others suggest this is low. Asparagus is a rich source of flavonoids, mainly rutin and quercetin, glutathione and vitamins (vitamin C and E) (Albanese et al., 2007). Most phenolic compounds are in violet (pale-colored) and green asparagus. White asparagus can be considered as a vegetable with a low antioxidant potential (Papoulias et al., 2009). This means that exposure to light is essential for the accumulation of phenolic compounds and that these compounds are accumulated in asparagus. Cooked asparagus had a higher antioxidant activity ( $20 \%$ increase) than raw asparagus (Papoulias et al., 2009). The purpose of this study was to examine the antioxidant potential and polyphenol composition of white, pale-colored and green asparagus spears of different cultivars.

\section{MATERIAL AND METHODS}

\section{Asparagus samples}

Five varieties of asparagus (Schwetzinger Meisterschuss, Huchel Alpha, Gimlin, Grolim and Eposs) were analysed. The material was collected in Nowy Tomyśl, Poland (Miedzichowo). Previously cleaned asparagus was stored in the refrigerator before heat treatment. The asparagus was cut into three-centimeter sections and boiled in water to soften it (about 20 minutes), and then after cooling the sample was homogenized. Asparagus was cut into a uniform particle size in order to unify the conditions for heat treatment. The asparagus was boiled to observe the chemical composition and antiooxidant activity of asparagus spears which are used for human consumption. Next, the samples were lyophilized (CHRIST 1-4 LSC, Germany) by applying constant parameters under the following conditions: temperature on the freeze dryer shelf $+20^{\circ} \mathrm{C}$, in the product $+4^{\circ} \mathrm{C}$, condensation temperature $-48^{\circ} \mathrm{C}$, at reduced pressure. The freeze drying time was approximately $48 \mathrm{~h}$. The sample prepared in this way was packed in dark glass and stored at room temperature.

The samples were defined and labeled as follows: GRw - white asparagus Grolim, GRp - pale-colored asparagus Grolim, GRg - green asparagus Grolim, $\mathrm{SCw}$ - white asparagus Schwetzinger Meisterschuss, $\mathrm{SCp}$ - pale-colored asparagus Schwetzinger Meisterschuss, $\mathrm{SCg}$ - green asparagus Schwetzinger Meisterschuss, GYw - white asparagus Gyjmlin, Gyp - pale-colored asparagus Gyjmlin, GYg - green asparagus Gyjmlin, EPw - white asparagus Eposs, EPp - pale-colored asparagus Eposs, EPg - green asparagus Eposs, HUw - white asparagus Huchel's Alpha, HUp - pale-colored asparagus Huchel's Alpha, Hug - green asparagus Huchel's Alpha. 


\section{Chemicals}

Chemicals such as 2,2-diphenyl-1-picrylhydrazyl (DPPH), 6-hydroxy-2,5,7,8-tetramethylchroman-2-carboxylic acid (Trolox), 2,2'-azinobis-(3-ethylbenzothiazoline-6-sulfonic acid) (ABTS) were purchased from Sigma-Aldrich (St. Louis, MO, USA). Flavonol and phenolic acid standards included quercetin, isoquercetin, kaempferol, hyperoside gallic caffeic acid, chlorogenic acid, p-coumaric acid, ferulic acid and sinapic acid - all were of HPLC-grade purity and purchased from Sigma-Aldrich Chemical Co. (St Louis, MO, USA).

\section{Preparation of crude extracts}

$5 \mathrm{~g}$ of lyophilized asparagus were extracted with $70 \mathrm{ml}$ of $60 \%$ ethanol for $2 \mathrm{~h}$ at room temperature according to the methodology described by Kobus et al. (2009) with a slight modification. Next, the supernatant was filtered using Whatman No 4 paper and rinsed with $50 \mathrm{ml}$ of ethanol. The extraction procedure was repeated according to the same conditions. Total supernatant was evaporated under vacuum conditions at $40^{\circ} \mathrm{C}$ and residual water was removed by liofilization to produce dry extract. Thus the resulting crude extracts were stored in a dry, dark and cool place until they were analyzed.

\section{Total polyphenolic content}

Total phenolic content (TPC) in the crude extracts of asparagus was established using UV-VIS with a colorimetric oxidation/reduction reaction was determined according to Cheung et al. (2003). A mixture of methanol and water $(1: 1, \mathrm{v} / \mathrm{v})$ was used to dissolve dry crude extracts. Next, a series of test tubes were supplemented with $1 \mathrm{~mL}$ of this solution. One milliliter of Folin-Ciocalteu's phenol reagent was added to individual test tubes and vortexed for $\sim 60 \mathrm{~s}$. One milliliter of a $35 \%(\mathrm{w} / \mathrm{v}) \mathrm{NaHCO}_{3}$ solution was added and the mixture was left in the dark for $90 \mathrm{~min}$ to ensure maximum color development. The samples were centrifuged at 3000× g (Sigma 204 laboratory centrifuge, St. Louis, MO, USA) before absorbance measurements were made. Absorbance of the supernatant was recorded at $\lambda=725 \mathrm{~nm}$ using a Meterek SP 830 apparatus (Taiwan). TPC values in asparagus extracts were given as $\mathrm{mg}$ quercetin equivalents/100 $\mathrm{g}$ dry asparagus using a plotted standard curve.

\section{Composition of phenolic acids}

The procedure was based on the methodology published by Kobus et al. (2009). The phenolic acids were analyzed as a percentage composition using Agilent UPLC equipped with a Bin Pump Infinity DAD 1290 detector $(\lambda=260 \mathrm{~nm}$ and $310 \mathrm{~nm})$. A non-linear concentration gradient was used to analyze the dry extract. The gradient consisted of $\mathrm{H}_{3} \mathrm{PO}_{4}$ buffer (solvent $\mathrm{A}), \mathrm{pH}=2.7$, which was adjusted using $1: 1 \mathrm{v} / \mathrm{v}$ acetonitrile-water (solvent B). The gradient profile decreased from $95 \%$ of solvent A at $1 \mathrm{~min}$ to $50 \%$ of B at $52 \mathrm{~min}$ and the run time was $58 \mathrm{~min}$. The volume of samples after filtration (PTFE filter; $0.45 \mu \mathrm{m}$ ) was $10 \mu 1$. Phenolic acids were identified using the standards dissolved in methanol (caffeic, chlorogenic, ferulic, p-coumaric and sinapic acids).

\section{Composition of flavonols}

The percentage composition of flavonols was determined according to the method described by Kobus et al. (2009). Extracted flavonols were separated and identified by Agilent UPLC using a Nova-Pak C18 reversed-phase column $(3.9 \times 150 \mathrm{~mm}, 5-\mu \mathrm{m}$ particle size; both from Waters, Milford, MA, USA). Solvent A was $0.3 \%(\mathrm{v} / \mathrm{v}) \mathrm{HCOOH}$ in $\mathrm{H}_{2} \mathrm{O}$, while solvent $\mathrm{B}$ was $100 \% \mathrm{CH}_{3} \mathrm{CN}$. The flow rate was maintained at $1 \mathrm{~mL} / \mathrm{min}$. The gradient profile was as follows: $85 \%$ of $\mathrm{A}$ at $0 \mathrm{~min}$ and $25 \%$ of $\mathrm{A}$ at $40 \mathrm{~min}$. Chromatograms were recorded using a UV-Vis detector at $\lambda=370 \mathrm{~nm}$. Separated compounds of individual flavonols were determined as isoquercetin, quercetin, hyperoside and kaempferol and their percentage composition was calculated.

\section{Antiradical activity with DPPH}

Crude extracts were assessed in a methanolic solution of $\mathrm{DPPH}^{*}$ to evaluate their free-radical scavenging potentials according to the method described by Amarowicz et al. (2006). The degree of solution discoloration indicated the scavenging efficacy of the added substance. One milliliter of the extract solution was supplemented with $2 \mathrm{~mL}$ of $\mathrm{CH}_{3} \mathrm{OH}$, followed by $0.25 \mathrm{~mL}$ of a $1-\mathrm{mM} \mathrm{DPPH}{ }^{*}$ ethanolic solution. The mixture was vortexed for $\sim 60 \mathrm{~s}$ and left for $20 \mathrm{~min}$ at room temperature. Absorbance was recorded at $\lambda=517 \mathrm{~nm}$ (Meterek SP 830, Taiwan). Methanol was 
used to prepare a reference sample and the control. To plot a calibration curve, absorbances were measured simultaneously in samples containing respective concentrations of the standard (Trolox 0.5, 1.0, 1.5 and $2.0 \mathrm{mg} / \mathrm{mL}$ ). The results were expressed as $\mathrm{mg}$ Trolox equivalents/g d.w. extract.

\section{Antiradical activity with ABTS}

A ABTS radical cation decolorization assay was used to estimate Trolox equivalent antioxidant capacity (TEAC) of asparagus extracts according to Re et al. (1999). Aqueous stock solutions of ABTS (7 mM) and potassium peroxodisulfate $(140 \mathrm{mM})$ were prepared and mixed to a final concentration of $2.45 \mathrm{mM}$ potassium peroxodisulfate. The mixture was left in the dark at room temperature for $12-16 \mathrm{~h}$ to react. On the day of analysis the ABTS radical solution was diluted with ethanol to provide absorbance amounting to 0.70 \pm 0.02 at $734 \mathrm{~nm}$. All measurements were conducted in the following manner: $100 \mu \mathrm{l}$ of asparagus extract was supplemented with $2.0 \mathrm{ml}$ of the ABTS radical solution, and absorbance values were recorded after precisely 6 min against the respective reagent blank of $100 \mu 1$ ethanol instead of the sample. The results from triplicate analyzes, derived from a calibration curve determined for this standard $(100-1000 \mu \mathrm{M})$, were expressed as Trolox equivalents/g d.w. extract.

\section{Chelating activity}

Chelating activities of crude asparagus extracts were measured according to Tang et al. (2002). In the colorimetric assay, the amount of unchelated $\mathrm{Fe}^{2+}$ in the crude asparagus extract after its reaction with 3-(2-pyridyl)-5,6-bis(4-phenylsulfonic acid)-1,2,4-triazine monosodium salt (i.e., ferrozine) was determined. One milliliter of the sample, $0.1 \mathrm{~mL}$ of $2 \mathrm{mM}$ $\mathrm{FeCl}_{2}$ and $0.2 \mathrm{~mL}$ of ferrozine reagent were added to each tube. The mixture was vortexed for $\sim 60 \mathrm{~s}$ and left for $20 \mathrm{~min}$ at room temperature. Absorbance values were recorded $(\lambda=562 \mathrm{~nm})$ using the Meterek SP 830 apparatus (Taiwan). Deionized water was used as a control and ferrozine as a reference. The chelating activity was calculated according to the following formula:

Chelating activity $=1-[($ Abs of sample - Abs of reference)/Abs of control) $] \times 100$
Experimentally determined $2000 \mathrm{ppm}$ asparagus extract concentrations were assessed.

\section{Statistical analysis}

All experiments were carried out in triplicate. The data obtained were subjected to statistical analysis for correlation (Pearson correlation) and significance of differences (post-hoc test) $(p<0.05)$ STATISTICA ${ }^{\text {TM }} \mathrm{PL}$ 9.0 StatSoft).

\section{RESULTS AND DISCUSSION}

\section{The yield and total polyphenols}

Data concerning the yield and total polyphenols for different extracts of five cultivars of white, pale-colored (purple) and green asparagus are shown in Table 1. Following thermal processing, comminution and freeze drying, the material was extracted with an aqueous ethanol solution. This process produced an extract that comprised all the components resulting from the complete extraction process and which are non-volatile up to the boiling point of the solvent (Hromadkova et al., 1999). In this study, efficiencies of extraction processes were determined for soluble components from five asparagus cultivars depending on the cultivation conditions (white, pale-colored and green) using a 60\% aqueous ethanol solution. Ethanol is a polar proton solvent used as an extractant in the food industry. Moreover, as reported in the literature, it is extensively used as an extractant in studies on the antioxidant activity of various plant materials. Our study showed significant differences between samples in the yield of the extraction process. The amounts of extractable components expressed as a percentage by weight of dried asparagus ranged from $7.98 \%$ (Schwetzinger Meisterschuss, pale-colored) to $11.84 \%$ (Huchel's Alpha, white), respectively, while the yields from asparagus of the same color ranged from 10.11 to $10.84 \%$ in all white asparagus samples, from $7.98 \%$ to $8.87 \%$ in pale-colored asparagus samples and from $9.11 \%$ to $10.87 \%$ in green asparagus, respectively. It was seen that white asparagus is the most efficient source of extractable components.

The efficiency of the extraction processes is dependent on the properties of the compounds extracted, or their solubility in the solvent used (Rodríguez et al., 2005; Suhaj, 2006). According to the general principle 
Table 1. Extract yield and total phenolic contents of different asparagus samples

\begin{tabular}{lcc}
\hline Sample & $\begin{array}{c}\text { Yield } \\
\text { g/100 dry asparagus }\end{array}$ & $\begin{array}{c}\text { Total phenolics } \\
\mathrm{mg} / 100 \text { g d.w. of extract }\end{array}$ \\
\hline GRw & $10.11^{\mathrm{c}} \pm 0.02$ & $430^{\mathrm{c}} \pm 12$ \\
SCw & $10.12^{\mathrm{c}} \pm 0.01$ & $350^{\mathrm{b}} \pm 22$ \\
GYw & $10.14^{\mathrm{c}} \pm 0.03$ & $270^{\mathrm{a}} \pm 19$ \\
EPw & $11.79^{\mathrm{d}} \pm 0.02$ & $380^{\mathrm{b}} \pm 12$ \\
HUw & $11.84^{\mathrm{d}} \pm 0.04$ & $370^{\mathrm{b}} \pm 23$ \\
GRp & $8.18^{\mathrm{a}} \pm 005$ & $380^{\mathrm{b}} \pm 17$ \\
SCp & $7.98^{\mathrm{a}} \pm 0.03$ & $360^{\mathrm{b}} \pm 15$ \\
GYp & $8.45^{\mathrm{a}} \pm 0.02$ & $260^{\mathrm{a}} \pm 22$ \\
EPp & $8.87^{\mathrm{ab}} \pm 0.05$ & $420^{\mathrm{c}} \pm 29$ \\
HUp & $8.67^{\mathrm{a}} \pm 0.06$ & $340^{\mathrm{b}} \pm 14$ \\
GRg & $9.22^{\mathrm{d}} \pm 0.06$ & $730^{\mathrm{f}} \pm 34$ \\
SCg & $9.11^{\mathrm{b}} \pm 0.02$ & $590^{\mathrm{de}} \pm 31$ \\
Gyg & $10.34^{\mathrm{c}} \pm 0.05$ & $450^{\mathrm{c}} \pm 21$ \\
EPg & $10.67^{\mathrm{c}} \pm 0.03$ & $650^{\mathrm{e}} \pm 45$ \\
Hug & $10.87^{\mathrm{c}} \pm 0.02$ & $540^{\mathrm{d}} \pm 41$ \\
\hline
\end{tabular}

All abbreviations are defined in Material and methods - asparagus samples.

The results are mean values of three determinations \pm standard deviation.

Values sharing the same letter in a column are not significantly different $(P \leq 0.05)$.

of solubility, "like dissolves like", which means that chemical compounds having hydrogen atoms or hydroxyl groups will dissolve more readily in protic rather than aprotic solvents. The capacity of a chemical compound to penetrate the solution results from its asymmetrical structure, with a partly displaced electric charge. For this reason, as a result of the extraction of an organic material using a solvent such as aqueous ethanol solution, the following compounds will penetrate the extract: monosaccharides, oligosaccharides, organic acids, pigments, tannins and polyphenols. Quantitative proportions of these components may vary depending on the solvent used. Moreover, it may be assumed that a higher efficiency of extraction from green asparagus was probably connected with photosynthetic processes and greater contents, e.g. of polyphenolic compounds. Hydrolysis could also have occurred in forms connected with such polyphenolics as esters or glycosides in this asparagus. This was all manifested in the increased solubility of the components and as a result, higher extraction efficiency. In contrast, the parts of white asparagus growing underground probably contain smaller amounts of polyphenolic compounds and others produced by photosynthesis, while they contain more carbohydrates, including storage sugars. The results of this analysis are consistent with those reported in other studies (Liu et al., 2012). In samples of cultivars of white, pale-colored and green asparagus, the total contents of polyphenolic compounds were determined using Folin-Ciocalteau's reagent. It was found that ethanol asparagus extracts contained polyphenols and their amounts differed to a statistically significant degree, depending on the cultivar and color of asparagus. Among the asparagus samples analyzed, the highest amount of polyphenolic compounds were detected in the Grolim green asparagus cultivar (GRg $-730 \mathrm{mg} / 100 \mathrm{~g} \mathrm{d.w}$.), while the lowest amounts were contained in pale-colored cv. Gyjmlin (GYp). The highest amount of polyphenolics were present in green asparagus $(450-730 \mathrm{mg} / 100 \mathrm{~g}$ d.w.), while in white and pale-colored asparagus polyphenols were found at lower, comparable levels. The total amount of polyphenolics in white and pale-colored asparagus varied significantly, depending on the cultivar (260-430 mg/100 g d.w.). The total content of polyphenolics in asparagus has been investigated by many authors and the results of their studies may not be easily compared to those presented in this paper due to the varied approaches and methods applied in sample preparation and in calculating the results. In their study, Papoulias et al. (2009) determined polyphenolic contents in white asparagus at $0.33 \mathrm{mg} / 100 \mathrm{~g}$ d.m., while Sun et al. (2007a) reported $4.9 \mathrm{mg} / \mathrm{g}$ d.m. of white asparagus (as expressed in catechin equivalents). In turn, Gębczyński (2007) reported that fresh green asparagus contained $95.6 \mathrm{mg}$ polyphenolics in $100 \mathrm{~g}$ (expressed in chlorogenic acid equivalents), while after cooking it was $84.8 \mathrm{mg}$ in $100 \mathrm{~g}$. Lu et al. (2007) determined the total contents of phenolic compounds as converted to gallic acid equivalents. The values fell within the range of $0.7-1.62 \mathrm{mg}$ gallic acid $/ \mathrm{g}^{-1}$ fresh product. 


\section{Flavonols and phenoloc acids composition}

The present research included analysis of the percentage composition of selected flavonols and phenolic acids in cultivars of white, pale-colored and green asparagus. The results of this analysis are shown in Figure 1 and Figure 2. It was found that both the color of asparagus and the cultivar had a significant effect on the composition of phenolic acid and flavonols.

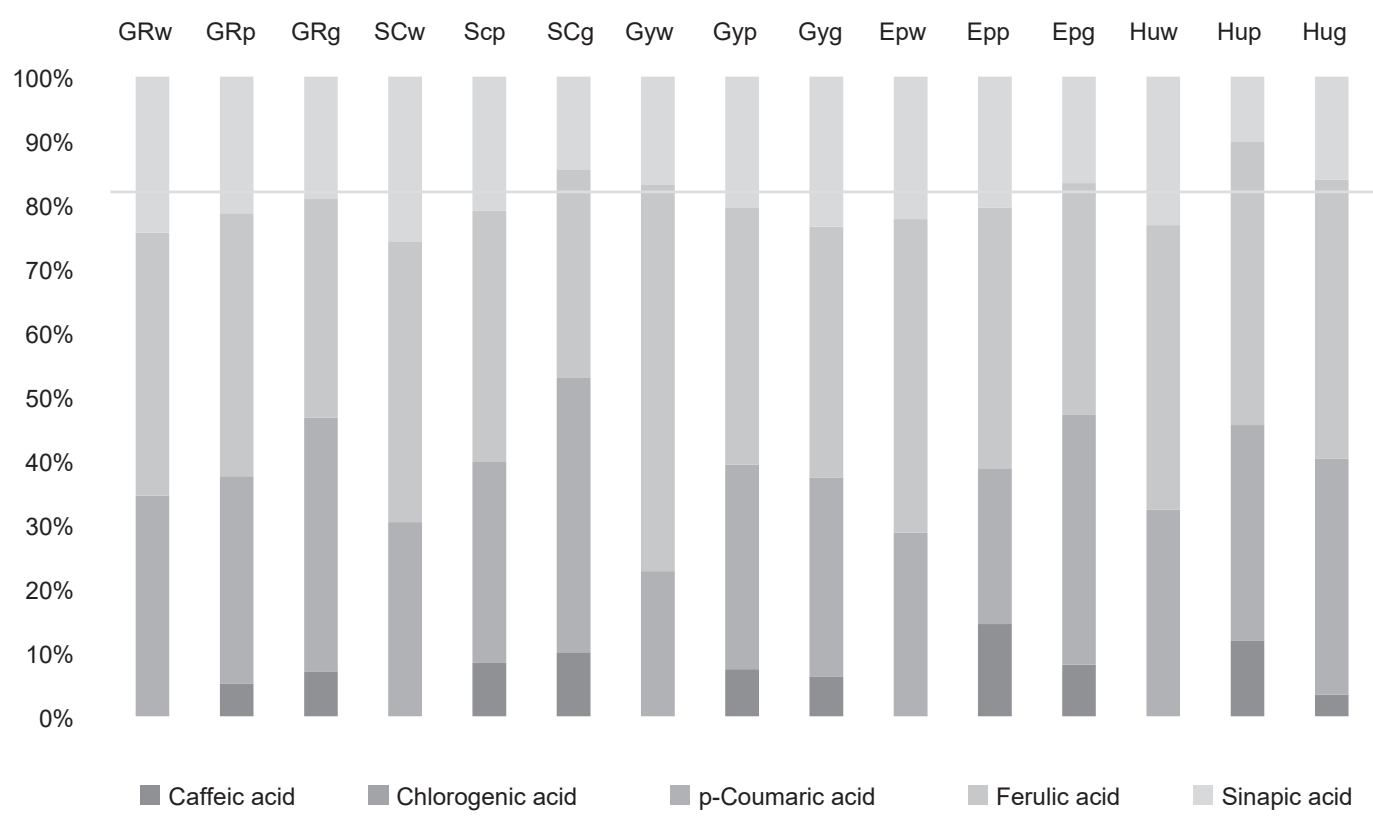

Fig. 1. The percentage composition of phenolic acids in asparagus from different cultivars

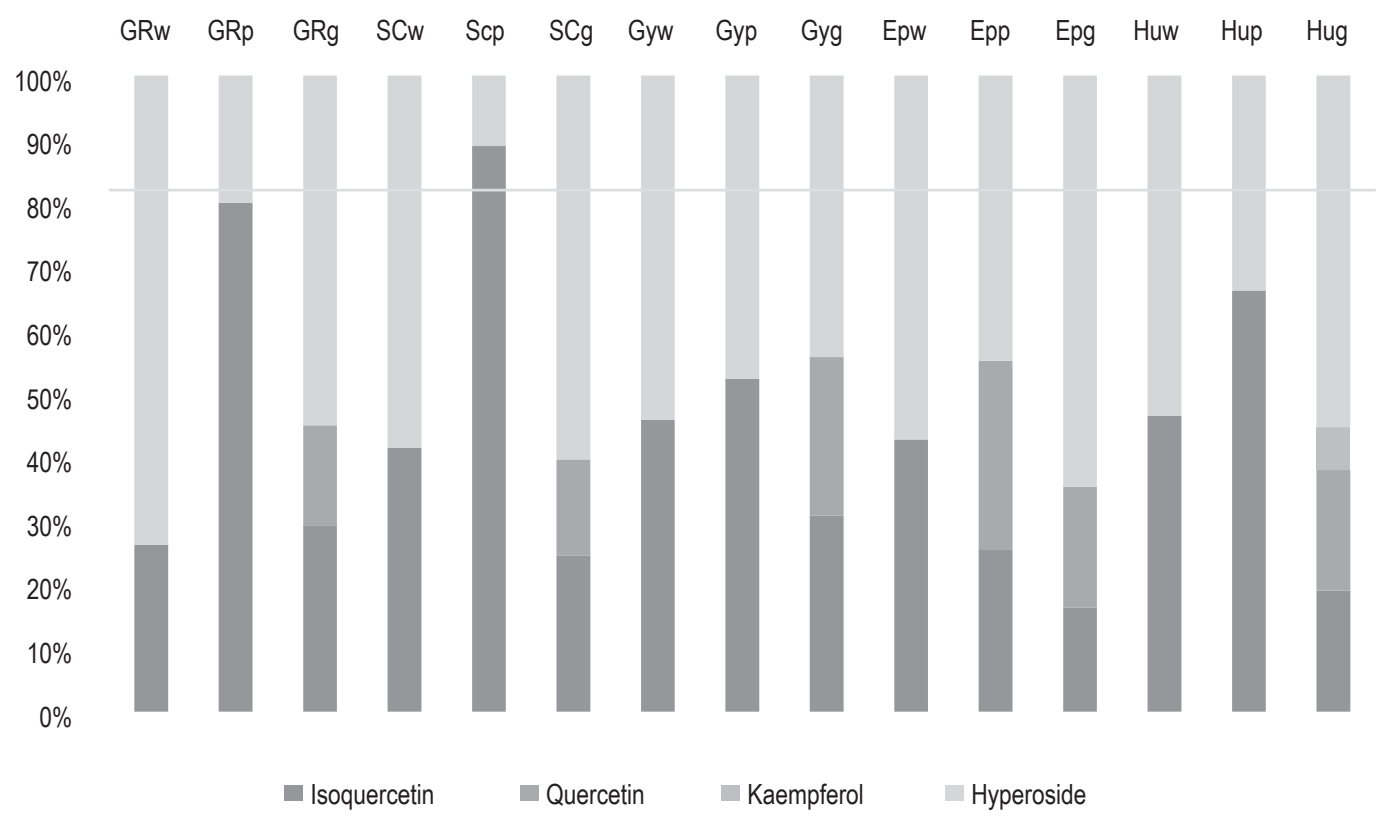

Fig. 2. The percentage composition of flavonols in asparagus from different cultivars 
Kulczyński, B., Kobus-Cisowska, J., Kmiecik, D., Gramza-Michałowska, A., Golczak, D., Korczak, J. (2016). Antiradical capacity and polyphenol composition of asparagus spears varieties cultivated under different sunlight conditions. Acta Sci. Pol. Technol. Aliment., 15(3), 267-279. DOI: 10.17306/J.AFS.2016.3.26

Among the phenolic acids, the predominant ones were p-coumaric and ferulic, while either no or low levels (chlorogenic and caffeic acid) in all analysed asparagus, regardless of the variety and color, were evaluated. Moreover, sinapic acid was determined as the smallest percentage of the acids occurring in all varieties and color of asparagus.

In all boiled asparagus samples HPLC was used to determine the percentage composition of the following flavonols: isoquercetin, quercetin, kaempferol and hyperoside. It was shown that all the cultivars were characterized by a great variation in flavonol content (2).

Among the flavonols the predominant ones were isoquercetin and hyperoside. Quercetin and kempferol in all analysed asparagus, regardless of the variety and color, were estimated on no or low levels. It has been clarified that a change in flavonol composition and increase in their diversity depends on them growing with sunlight, and was higher for green asparagus and lower for white asparagus. The varied qualitative composition of phenolic acids and flavonols resulted most probably from changes occurring during vegetation, such as a lack of access to light in the case of white asparagus and limited access to light in pale-colored asparagus. Furthermore, the cultivar of asparagus had a significant effect on the amounts of this compounds. Similar results were reported by Fuentes-Alventosa et al. (2009). The authors reported differences in flavonoid composition in 32 cultivars of asparagus. Similarly, the effect of asparagus color on the composition of polyphenols was investigated by Guillén et al. (2008). Those authors reported that green asparagus contains greater amounts of flavonoids and hydroxycinnamic acids. Shou et al. (2007) also reported that vegetables grown in the shade contained less polyphenols, rutin, ascorbic acid and chlorophyll than asparagus grown in sunlight.

\section{Antioxidant activity of asparagus with DPPH radicals}

Antioxidant activity of extracts from the asparagus samples tested was determined with radical DPPH (1,1-diphenyl-2-picrylhydrazyl). The results are expressed as Trolox equivalent (mmol Trolox/g extract d.m) and are given in Table 2. It was found that all these asparagus extracts had the capacity to neutralize free radical DPPH*. The activity of the extracts
Table 2. Antioxidant activity of asparagus extract with DPPH and ABTS radicals

\begin{tabular}{lcc}
\hline Sample & $\begin{array}{c}\text { DPPH } \\
\text { mmol Trolox/g extract } \\
\text { d.m. }\end{array}$ & $\begin{array}{c}\text { ABTS } \\
\text { mmol Trolox/g extract } \\
\text { d.m. }\end{array}$ \\
\hline GRw & $1.22^{\mathrm{b}} \pm 0.02$ & $0.84^{\mathrm{b}} \pm 0.01$ \\
SCw & $0.34^{\mathrm{a}} \pm 0.01$ & $0.43^{\mathrm{ab}} \pm 0.03$ \\
GYw & $2.02^{\mathrm{c}} \pm 0.01$ & $0.13^{\mathrm{a}} \pm 0.03$ \\
EPw & $0.58^{\mathrm{a}} \pm 0.01$ & $0.40^{\mathrm{ab}} \pm 0.02$ \\
HUw & $1.49^{\mathrm{b}} \pm 0.05$ & $0.52^{\mathrm{b}} \pm 0.02$ \\
GRp & $0.87^{\mathrm{ab}} \pm 0.03$ & $0.37^{\mathrm{a}} \pm 0.01$ \\
SCp & $0.41^{\mathrm{a}} \pm 0.01$ & $0.38^{\mathrm{a}} \pm 0.02$ \\
GYp & $1.71^{\mathrm{b}} \pm 0.02$ & $0.28^{\mathrm{a}} \pm 0.03$ \\
EPp & $1.40^{\mathrm{ab}} \pm 0.01$ & $0.57^{\mathrm{b}} \pm 0.02$ \\
HUp & $1.61^{\mathrm{b}} \pm 0.01$ & $0.33^{\mathrm{a}} \pm 0.01$ \\
GRg & $3.45^{\mathrm{d}} \pm 0.05$ & $2.41^{\mathrm{ab}} \pm 0.45$ \\
SCg & $2.47^{\mathrm{c}} \pm 0.01$ & $1.92^{\mathrm{d}} \pm 0.03$ \\
GYg & $4.49^{\mathrm{e}} \pm 0.02$ & $1.49^{\mathrm{c}} \pm 0.03$ \\
EPg & $5.18^{\mathrm{e}} \pm 0.05$ & $1.76^{\mathrm{cd}} \pm 0.02$ \\
HUg & $3.84^{\mathrm{d}} \pm 0.03$ & $1.96^{\mathrm{d}} \pm 0.01$ \\
\hline & &
\end{tabular}

All abbreviations are defined in Material and methods - asparagus samples.

The results are mean values of three determinations \pm standard deviation.

Values sharing the same letter in a column are not significantly different $(P \leq 0.05)$.

ranged from 0.34 for $\mathrm{SCw}$ samples to $5.18 \mathrm{mmol}$ Trolox/g extract d.m. for EPg samples. It was seen that green asparagus exhibits a statistically significant higher scavenging capacity in relation to the $\mathrm{DPPH}^{\circ}$ radical (2.47-5.18) than white asparagus $(0.34-2.02$ mmol Trolox/g extract d.m.) or pale-colored asparagus $(0.87-1.71 \mathrm{mmol}$ Trolox/g extract d.m.). The activity of white asparagus was lowest and ranged from 0.34 mmol Trolox for $\mathrm{SCw}$ to BE 2.02 mmol Trolox for GYw. No evident dependence was observed between antioxidant activity and asparagus cultivar, while it was seen that the color of asparagus (white, pale-colored and green) had a significant effect on radical scavenging activity. 
Kulczyński, B., Kobus-Cisowska, J., Kmiecik, D., Gramza-Michałowska, A., Golczak, D., Korczak, J. (2016). Antiradical capacity and polyphenol composition of asparagus spears varieties cultivated under different sunlight conditions. Acta Sci. Pol. Technol. Aliment., 15(3), 267-279. DOI: 10.17306/J.AFS.2016.3.26

Plants and extracts prepared from them are composed of numerous metabolites that may behave as agonists or antagonists in radical scavenging activity (Gramza-Michalowska et al., 2011; Kobus-Moryson and Gramza-Michałowska, 2015). However, Sun et al. (2007a) reported that antioxidant capacity in many plant materials is connected primarily with their polyphenol contents, indicating a significant positive correlation between total phenolic contents and antioxidant capacity. The same correlations were found in the present study. The results presented here are consistent with the available literature data. Sun et al. (2007b) determined the scavenging potential of free DPPH radicals in methanol extracts of white asparagus and in the resulting juice. It was seen that the radical scavenging activity of the methanol extract from asparagus was $17.4 \mathrm{mmol}$ Trolox $/ \mathrm{kg}$ vegetable d.m., while for asparagus juice it was $1.2 \mathrm{mmol}$ Trolox/l juice and it was correlated with the content of polyphenolics and sterols found in asparagus. Similar conclusions were drawn by Fuentes-Alventosa et al. (2009), who investigated high dietary fiber powders obtained from asparagus by-products. They studied factors such as treatment intensity, the solvent used and the drying system. Moreover, they estimated the content of phytochemicals that were found in the samples. These included hydroxycinnamic acids, saponins, flavonoids, sterols and fructans. Samples were evaluated in terms of their activity toward DPPH radicals and it was found that the high activity of these samples in relation to the radicals was positively correlated with hydroxycinnamic acids and flavonoids. In a study by Papoulias et al. (2009), they found a high level of phytocompound activitys in asparagus, while also stating that thermal processing had no significant effect on the scavenging potential of free DPPH radicals. Similarly, Wang et al. (2011) showed that antioxidant activity was also exhibited by compounds contained in green asparagus. These authors determined the activity in juice from fresh green asparagus and the percentage level of radical scavenging depended on the concentration of prepared extracts, which was then correlated to polyphenolics, just as was done in this study. Morever, Kobus et al. (2009) indicated that antioxidant activity of extract from Ginkgo biloba depends on the contents and proportions of individual phytocompounds. Statistical analysis showed a significant positive correlation between total flavonol content and activity toward $\mathrm{DPPH}^{\cdot}(r=0.543)$. The presence of flavonols, such as quercetin $(r=0.781)$, kaempferol $(r=0.623)$ and morin $(r=0.552)$, contributed to the high antioxidant activity of Gingko biloba extract toward DPPH radicals.

The results of analyzes conducted in this study indicated the asparagus with the highest radical scavenging activity in relation to DPPH - green asparagus. The analyzes also made it possible to identify cv. Gyjmlin and Grolim as cultivars exhibiting the highest scavenging potential in relation to DPPH radicals.

\section{Antioxidant activity of asparagus with $A B T S$ radicals}

There are many analytical methods facilitating assessment of relative antioxidant potential or the capacity for simple substances and their mixtures. One of these is the method using the ABTS cation radical. Antioxidants found in the analyzed sample cause a reduction of cation radicals to a degree that is dependent on the duration of the reaction, antioxidant concentration and its activity. The solution color disappears, with the degree of color intensity being proportional to the content of antioxidants in the solution. Taking into consideration the definite effect of polyphenolics, including flavonols and phenolic acids, on antioxidant activity, measurements were taken for the capacity of binding free ABTS radicals by extracts from white, pale-colored and green asparagus cultivars: Grolim, Schwetzinger Meisterschuss, Gyjmlin, Eposs, Huchel's Alpha. The antioxidant capacity of extracts was expressed as activity in relation to a synthetic and water-soluble tocopherol analog-Trolox, and the results were expressed in mmol Trolox/g extract d.m. and are presented in Table 2. The activity of the asparagus tested, which was measured in a system with the ABTS radical, ranged from 0.13 to $2.41 \mathrm{mmol}$ Trolox/g extract d.m., with green asparagus exhibiting the highest activity, falling in the range from 1.49 for GYg samples to $2.41 \mathrm{mmol}$ Trolox/g extract d.m. for GRg samples. The activity of extracts produced from white and pale-colored asparagus was lower and ranged from 0.13 for GYw to $0.84 \mathrm{mmol}$ Trolox/g extract d.m. for GRw samples. It was found that the colors of both the asparagus and cultivar had a significant effect on the antiradical activity of the extracts $(p<0.05)$. Murcia 
Kulczyński, B., Kobus-Cisowska, J., Kmiecik, D., Gramza-Michałowska, A., Golczak, D., Korczak, J. (2016). Antiradical capacity and polyphenol composition of asparagus spears varieties cultivated under different sunlight conditions. Acta Sci. Pol. Technol. Aliment., 15(3), 267-279. DOI: 10.17306/J.AFS.2016.3.26

et al. (2009) investigated the antioxidant activity of raw, fresh and frozen asparagus compared to 25 other vegetables (artichoke, asparagus, beetroot, broad bean, broccoli, brussels sprout, carrot, cauliflower, celery, chicory, cucumber, eggplant, endive, garlic, green bean, leek, lettuce, maize, onion, pea, pepper, radish, spinach, Swiss chard and zucchini). Asparagus was classified to the group of vegetables with good antioxidant properties and their activity was ranked 10th. Moreover, it was seen that antioxidant activity during cold storage decreased, although the difference between the antioxidant potential of fresh and frozen vegetables was not statistically significant. In turn, Sun et al. (2007b) determined antioxidant potential in methanol extracts from asparagus. The values were given as mmol Trolox $/ \mathrm{kg}$ d.m. and were as follows: $27.1 \mathrm{mmol}$ Trolox per $1 \mathrm{~kg} \mathrm{~d}$.m., while in asparagus juice these values were lower, amounting to $3.3 \mathrm{mmol}$ Trolox/l juice.

It is assumed that antioxidant activity is generally connected with the content of polyphenolics, which has been confirmed by numerous other studies (Re et al., 1999; Sun et al., 2007b; Maeda et al., 2008; Murcia et al., 2009; Papoulias et al., 2009; Kmiecik et al., 2011; Liu et al., 2012; Kobus-Cisowska et al., 2014; Kmiecik et al., 2015) as well as the results of the present study. However, not all authors observed such a dependence. Heimler et al. (2005), when analyzing the level of polyphenolics and antioxidant activity in beans over a period of three years, stated that beans, which are richest in such polyphenolic compounds as proanthocyanidins and flavonoids, exhibited only medium antioxidant activity. The researchers were of the opinion that antioxidant activity is influenced by both the total amount of contained polyphenolics and the proportion of individual groups in the total pool of these substances. Hyun et al. (2006) showed that phenolic acids exhibit strong antiradical properties. However, most often their content is not correlated with the results of analysis of their antiradical effects. This fact results from the proportions at which they are found, primarily the structure of individual compounds (Gramza-Michałowska and Człapka-Matyasik 2007; Maeda et al., 2008; Natella et al., 1999; Wang et al., 2011). According to Kobus et al. (2009), $1 \mathrm{mmol}$ quercetin has an antioxidant activity of $4.7 \mathrm{mmol}$ Trolox, while for its glycoside form (isoquercitrin) it is only $2.7 \mathrm{mmol}$ and for rutin $2.4 \mathrm{mmol}$, respectively. Quercetin is considered to be one of the best scavengers of reactive oxygen species, since it is a flavonol, which contains a catechol group in the B-ring, a double $\mathrm{C}-2 \mathrm{C}-3$ bond and a hydroxyl group in position C-3. Moreover, it is assumed that the B-ring, spatially arranged properly in the molecule, and its slope in relation to the other part of the molecule may be crucial for efficient electron transfer and may determine its antioxidant properties (Cheung et al., 2003; Kohmura et al., 2008). When discussing the results of radical scavenging activity in relation to the ABTS cation radical, it needs to be stressed that often it may hardly have any reference to the data presented in the extensive literature on the subject because extracts tested by other authors exhibited activity expressed in various units, which was connected with the different methods applied. However, it may definitely be stated that the antioxidant activity of the asparagus was comparable to that of extracts from other plants with documented antioxidant potential. Kirakosyan et al. (2003) compared the activity of individual model polyphenolic solutions with the activity of ethanol extracts from hawthorn fruits. Those studies showed that extracts produced from hawthorn exhibited a very high capacity to inactivate ABTS cation radicals (radical scavenging activity of 2.3 mmol Trolox), almost 30\% greater than free (-) epicatechin. The authors proposed a hypothesis that such results suggest synergism of individual polyphenolics contained in the extract. In contrast, Natella et al. (1999) compared the antiradical action of certain polyphenolics, singly and in mixtures, in relation to ABTS cation radicals. Polyphenols tested separately and in mixtures had similar activities, and occasionally (in the case of cinnamic and caffeic acids) in the mixture they exhibited a slightly weaker action than singly. This may be explained by means of the complicated mechanisms taking place between individual polyphenolics, as well as between polyphenolics, and other components of the solutions tested. Prior to the analysis of the antiradical properties of five white, pale-colored and green asparagus cultivars in relation to ABTS, it was stated that green asparagus in the case of cultivation method and asparagus cv. Grolim exhibited the highest capacity to inactivate the ABTS cation radical. At the same time, it was seen that this activity was dependent on the contents of polyphenolic 
compounds, including the presence of isoquercetin $>$ rutin $>$ gallic acid $>$ ferulic acid.

\section{Assessment of chelating capacity of Fe $2+$ ions}

Ions of transition metals such as copper and iron participate in the oxidation process, since they are catalysts in the reaction of hydroperoxide degradation. As a result of hydrogen peroxide reduction, the most reactive hydroxyl radicals are generated (the Fenton and Haber Weiss reaction). The binding of ions into stable complexes called chelates had a significant effect on oxidation processes. The kinetics of this reaction consist in the attachment of a metal ion by the molecule (antioxidant) through a coordinate bond via the so-called donor atom, containing a free pair of electrons (Correia and McCue, 2004; Fernandez et al., 2002).

In samples of white, pale-colored and green asparagus cultivars of Grolim, Schwetzinger Meisterschuss, Gyjmlin, Eposs and Huchel's Alpha, the chelating capacity of two-valent metal ions (Fe 2+) was determined. Figure 3, Figure 4 and Figure 5 presents the

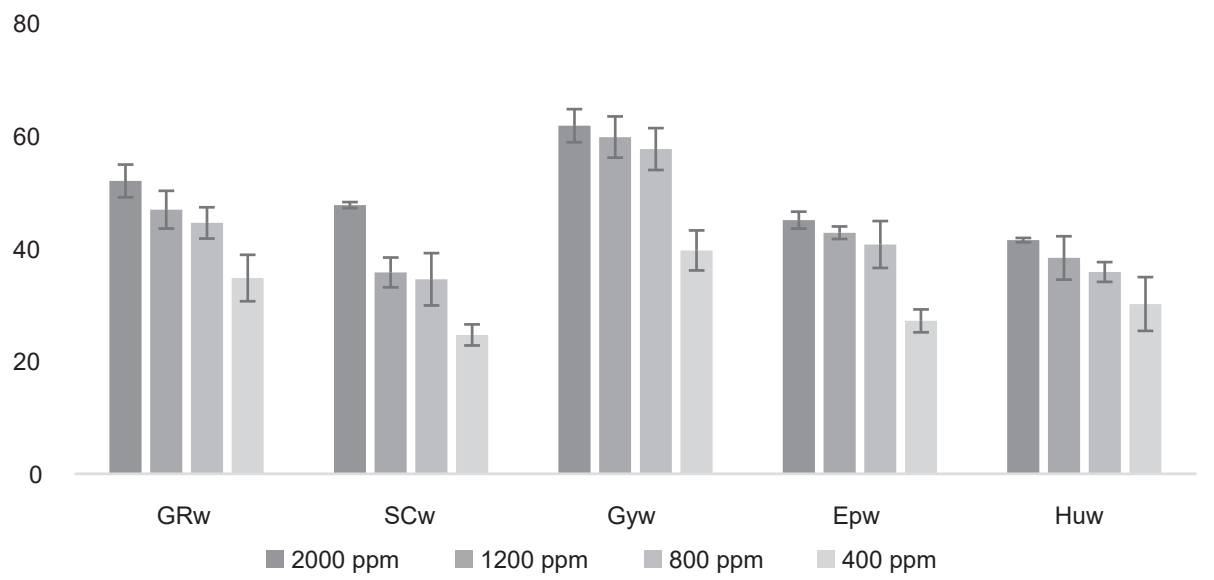

Fig. 3. The metal ion chelating activity of white asparagus, $\%$

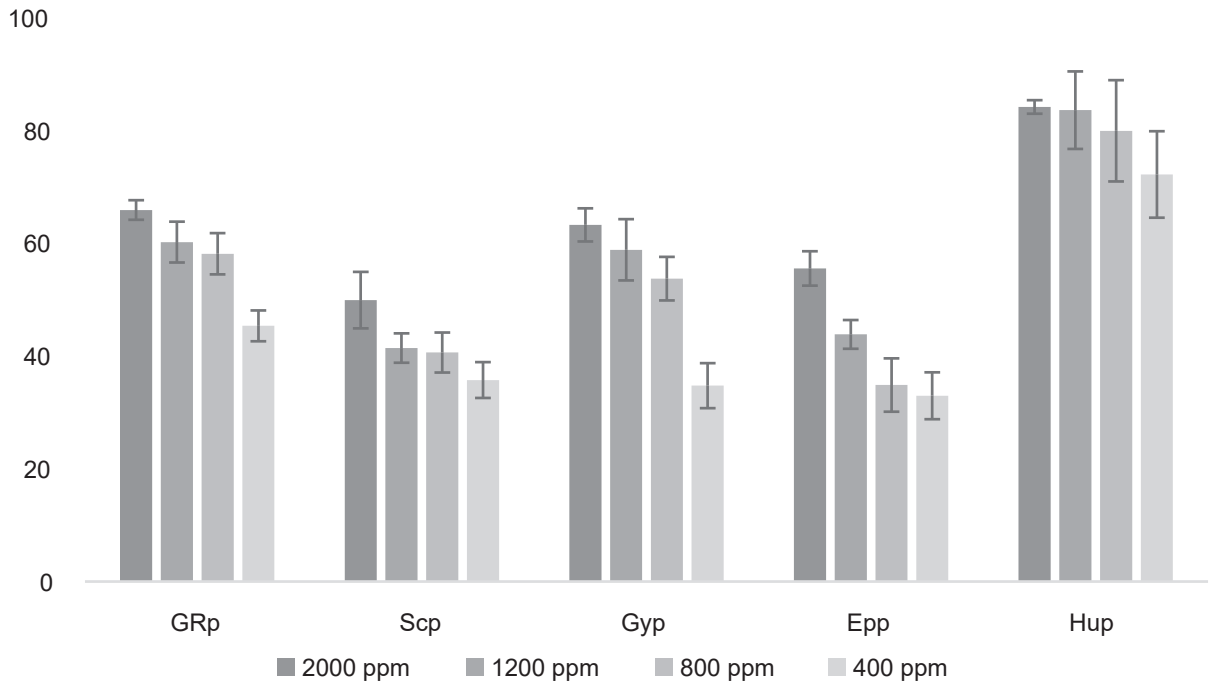

Fig. 4. The metal ion chelating activity of pale-colored asparagus, $\%$ 


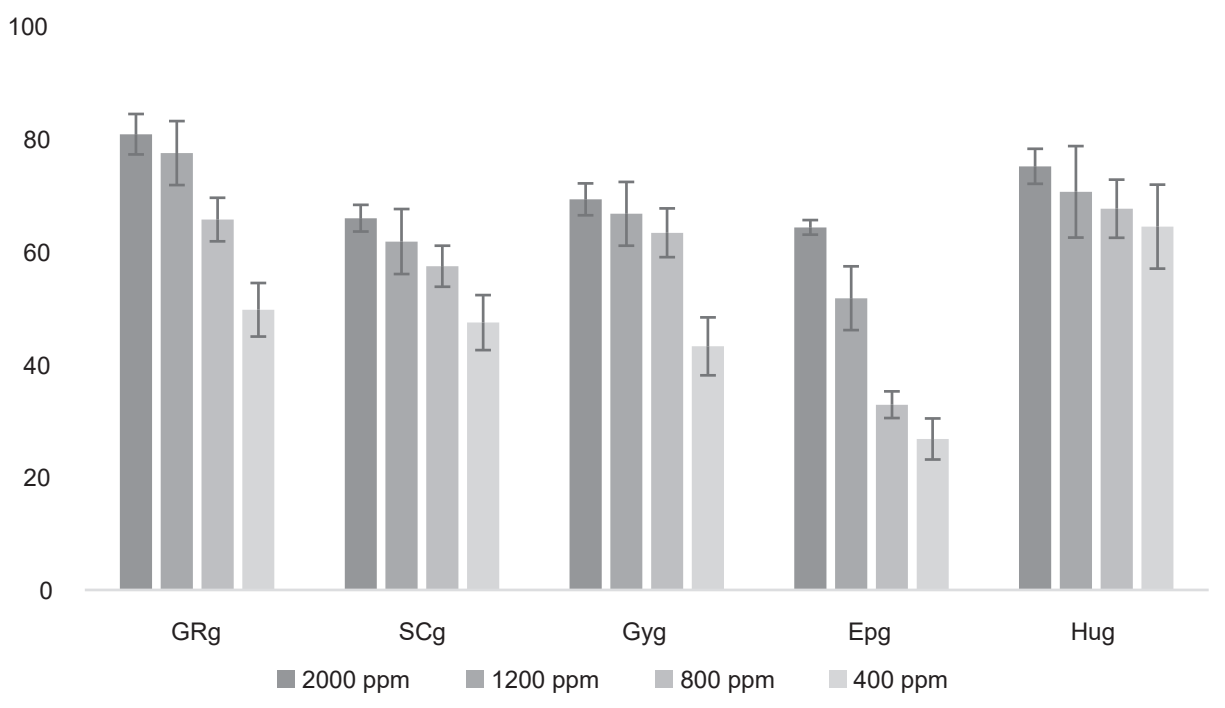

Fig. 5. The metal ion chelating activity of green asparagus, $\%$

results of analyzes of $2000 \mathrm{ppm}, 1200 \mathrm{ppm}, 800 \mathrm{ppm}$ and 400 ppm concentration of extract concerning the chelating activity expressed in percentage.

It was seen that all the asparagus samples, irrespective of the cultivar and color, exhibited chelating capacity. The greatest number of iron ions were chelated by samples of green asparagus, ranging from $44.33 \%$ in a concentration of $2000 \mathrm{ppm}$ for EPg to $60.87 \%$ for GRg, with the lower level for pale-colored asparagus samples ranging from $29.94 \%$ for SCp to $64.21 \%$ for $\mathrm{HUp}$, while the capacity was lowest in white asparagus, ranging from $31.98 \%$ to $41.78 \%$ for GYw. The chelating ability increases in the concentration of extracts from $400 \mathrm{ppm}$ to $800 \mathrm{ppm}$ and $1200 \mathrm{ppm}$ and then to $2000 \mathrm{ppm}$ in all analysed samples. It was also found that compounds contained in extracts from asparagus cv. Huchel's Alpha and Grolim formed chelates with iron ions to a greater degree than the compounds extracted from the other cultivars.

Studies on the capacity of phytochemicals in asparagus spears to form complexes with metals have also been conducted by other authors. In a study by Wang et al. (2011), the chelating properties of asparagus juice were also high. This property increased with an increasing concentration of the extract. These values at concentrations of $0.1-0.5 \mathrm{mg} / \mathrm{ml}$ fell within the range of $26-47 \%$. The capacity to form complexes with metals depends on the structure of phenolic compounds, primarily the amounts and position of hydroxyl groups. The chelating properties of compounds containing a greater number of functional groups are respectively stronger. The location of metal bonding to a flavonoid molecule in the ortho-diphenolic group is at the position 3',4' in the B-ring and in the C-ring for the 4-keto and 3-hydroxy or 4-keto and 5-hydroxy groups (Pereira et al., 2009).

\section{CONCLUSIONS}

It was seen that asparagus spears presented varied activity to scavenge radicals. The Grolim and Gymlin cultivars showed the highest activity in DPPH radical and ABTS cation radical free radical tests. Their high capacity to form complexes with iron(II) was also observed. Much greater antioxidant properties were found in green asparagus when compared to white and pale-colored asparagus, with the activity resulting from the presence of polyphenolic compounds. It was found that both the color of asparagus and the cultivar had a significant effect on composition of phenolic acid and flavonols. It has been clarified that changing flavonol and phenolic acid composition and increasing their diversity depends on them growing with sunlight, and was higher for green asparagus and lower for white asparagus. 
Kulczyński, B., Kobus-Cisowska, J., Kmiecik, D., Gramza-Michałowska, A., Golczak, D., Korczak, J. (2016). Antiradical capacity and polyphenol composition of asparagus spears varieties cultivated under different sunlight conditions. Acta Sci. Pol. Technol. Aliment., 15(3), 267-279. DOI: 10.17306/J.AFS.2016.3.26

\section{REFERENCES}

Albanese, D., Russo, L., Cinquanta, L., Brasiello, A., Di Matteo, M. (2007). Physical and chemical changes in minimally processed green asparagus during cold-storage. Food Chem., 101, 1, 274-280.

Amarowicz, R, Żegarska, Z., Pegg, R. B., Karamać, M., Kosińska, A. (2006). Antioxidant and radical scavenging activities of a barley crude extract and its fractions. Czech J. Food Sci., 25, 2, 73-80.

Cheung, L., Cheung, P., Ooi, V. (2003). Antioxidant activity and total phenolic of edible mushrooms extracts. Food Chem., 81, 249-255.

Correia, R., McCue, P. (2004). Production of phenolic antioxidants by solid-state bioconversion ofpineapple waste mixed with soy flour using Rhizopus oligosporus. Process Biochem., 39, 2167-2172.

Fernandez, T., Lurdes, M., Florêncio, M., Jennings, R. (2002). Iron and copper chelation by flavonoids, an electrospray mass spectrometry study. J. Inorg. Biochem., 92, 2, 105-111.

Fuentes-Alventosa, J., Jaramillo-Carmona, S., Rodríguez-Gutiérrez, G., Guillén-Bejarano, R., Jiménez-Araujo, A., Fernández-Bolaños, J., Rodríguez-Arcos, R. (2013). Preparation of bioactive extracts from asparagus byproduct. Food Bioprod. Process., 91, 2, 74-82.

Fuentes-Alventosa, J. M., Jaramillo-Carmona, S., Rodriguez-Gutierrez, G., Rodriguez-Arcos, R., Fernandez-Bolanos, J., Guillen-Bejarano, R., Espejo-Calvo, J. A., Jimenez-Araujo, A. (2009). Effect of the extraction method on phytochemical composition and antioxidant activity of high dietary fibre powders obtained from asparagus by-products. Food Chem., 116, 2, 484-490.

Gębczyński, P. (2007). Content of selected antioxidative compounds in Green asparagus depending on processing before freezing and on the period and conditions of storage. Pol. J. Food Nutr. Sci., 57, 2, 209-214.

Gramza-Michalowska, A., Sidor, A., Hes, M. (2011). Herb extract influence on the oxidative stability of selected lipids. J. Food Biochem., 35, 6, 1723-1736.

Gramza-Michałowska, A., Człapka-Matyasik, M. (2011). Evaluation of the antiradical potential of fruit and vegetable snacks. Acta Sci. Pol. Technol. Aliment., 10(1), 63-72.

Gramza-Michalowska, A., Korczak, J., Regula, J. (2007). Use of plant extracts in summer and winter season butter oxidative stability improvement. Asia Pac. J. Clin. Nutr. 16 (Supl. 1), 85-88.

Guillén, R., Rodríguez, R., Jaramillo, S., Rodríguez, G., Espejo, J. A., Fernandez- Bolaños, J., ..., Jiménez, A. (2008).
Antioxidant from asparagus spear: Phenolics. Acta Hort., 776, 247-254. DOI 10.17660/ActaHortic.2008.776.31.

Heimler, D., Vignolini, P., Dini, M., Romani, A. (2005). Rapid test to assess the antioxidant activity of Phaseolus vulgaris L., dry beans. J. Agric. Food Chem., 53, 3053-3056.

Hromadkova, Z., Ebringerova, A., Valachovic, P. (1999). Comparison of classical and ultrasoundassisted extraction of polysaccharides from Salvia officinalis L. Ultrason. Sonochem. 5, 163-168.

Hyun, S., Jung, H., Chung, H., Choi, J. (2006). In Vitro peroxynitrite scavenging activity of 6-Hydroxykynurenic acid and other flavonoids from Gingko biloba yellow leaves. Arch. Pharm. Res., 29, 12, 1074-1079.

Kirakosyan, A., Seymour, E., Kaufman, P., Warber, S., Bolling, S., Chang, S. (2003). Antioxidant capacity of polyphenolic extracts from leaves of Crataegus laevigata and Crataegus monogyna (hawthorn) subjected to drought and cold stress. J. Agric. Food Chem., 51, 14, 3973-3976.

Kmiecik, D., Korczak, J., Rudzińska, M., Gramza-Michałowska, A., Hęś, M., Kobus-Cisowska, J. (2015). Stabilisation of phytosterols by natural and synthetic antioxidants in high temperature conditions. Food Chem., 173, 966-971.

Kmiecik, D., Korczak, J., Rudzińska M., Kobus-Cisowska, J., Gramza-Michałowska, A., Hęś, M. (2011). B-Sitosterol and campesterol stabilisation by natural and synthetic antioxidants during heating. Food Chem., 128, 937-942.

Kobus, J., Flaczyk, E., Siger, E., Nogala-Kałucka, M., Korczak, J., Pegg, R. B. (2009). Phenolic compounds and antioxidant activity of extracts of Ginkgo biloba leaves. Eur. J. Lipid Sci. Tech., 111, 1150-1160.

Kobus-Cisowska, J., Flaczyk, E., Rudzińska, M., Kmiecik, D. (2014). Antioxidant properties of extracts from Ginkgo biloba leaves in meatballs. Meat Sci., 97, 2, 174-180.

Kobus-Moryson, M., Gramza-Michałowska, A. (2015). Directions on the use of stevia leaves (Stevia rebauidana) as an additive in food products. Acta Sci. Pol. Technol. Aliment., 14, 1, 5-13.

Kohmura, H., Watanabe, Y., Muto, N. (2008). Polyphenol content, antioxidant activity and surface colour of asparagus spears cultivated under different conditions of sunlight. Acta Hort., 776, 255-260.

Liu, Y., Qi, M., Li, T. (2012). Photosynthesis, photoinhibition, and antioxidant system in tomato leaves stressed by low night temperature and their subsequent recovery. Plant Sci., 196, 8-17. 
Lu, G., Shou, S., Huang, X. (2007). Seasonal valiatians in nutritional components of green asparagus rusing the mother fern cultivation. Sci. Hort., 112, 251-257.

Maeda, T., Kakuta, T. Sonoda, S. Motoki, K. Maekawa, Suzuki T., Oosawa K. (2008). Differences in varieties and seasonal change of antioxidative polyphenols contents in asparagus on various cultural conditions of the mother fern culture. Acta Hort., 776, 227-234.

Murcia, A., Jimenez, A., Martinez T. (2009). Vegetables antioxidant losses during industrial processing and refrigerated storage. Food Res. Int., 42, 1046-1052.

Natella, F., Nardini, M., Di Felice, M., Scaccini, C. (1999). Benzoic and cinnamic acid derivatives as antioxidants: structure-activity relation. J. Agric. Food Chem., 47, 4, 1453-1459.

Papoulias, E., Simons, A., Koukounaras, A., Gerasopoulos, D., Kazakis, E. (2009). Effect of genetic, pre- and postharvest factors on phenolic content and antioxidant capacity of white asparagus spears. Int. J. Mol. Sci., 10, 5371-5379.

Pereira, D. M., Valentao, P., Pereira, J. A., Andrade, P. B. (2009). Phenolics: from chemistry to biology. Molecules, 13, 2202-2211.

Re, R., Pellegrini, N., Proteggennte, A., Pannala, A., Yang, M., Rice-Evans, C. (1999). Antioxidant applying in improved ABTS radical cation decolorization assay. Free Radic. Biol. Med., 26, 9/10, 1231-1237.

Rodríguez, R., Jaramillo, S., Rodríguez, G., Espejo, Guillén, R., Fernandez- Bolaños, J., ..., Jiménez, A. (2005). Antioxidant activity of ethanolic extracts from several asparagus cultivars. J. Agric. Food. Chem., 53, 5212-5217.

Shou, S., Lu, G., Huang, X. (2007). Seasonal valiatians in nutritional components of green asparagus using the mother fern cultivation. Sci. Hort., 112, 251-257.

Suhaj, M. (2006). Spice antioxidants isolation and their antiradical activity, a review. J. Food Compost. Anal., 19, 531-537.

Sun, T., Powers, J. R., Tang, J. (2007a). Evaluation of the antioxidant activity of asparagus, broccoli and their juice. Food Chem., 105, 101-106.

Sun, T., Tang, J., Powers, J. (2007b). Antioxidant activity and quality of asparagus affected by microwave-circulated water combination and conventional sterilization. Food Chem., 100, 813-819.

Tang, S. Z., Kerry, J. P., Sheehan, D., Buckley, D. J. (2002). Antioxidative mechanism of tea catechins in chicken meat systems. Food Chem., 76, 45-51.

Wang, B., Chang, L., Wu, H., Huang, S., Chu, H., Huang, M. (2011). Antioxidant and antityrosinase activity of aqueous extract of green asparagus. Food Chem., 127, 141-146. 
